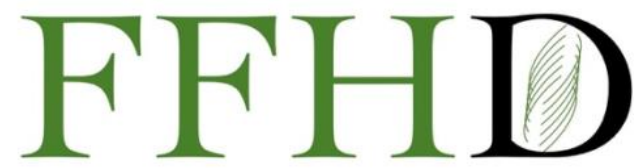

Functional Foods in Health and Disease

\title{
Suppressive effect of a single dose of monoglucosyl rutin on postprandial blood glucose elevation: A randomized, placebo- controlled, double-blind crossover study
}

\section{Yushi Hashizume $^{1^{*}}$, Mahamadou Tandia $^{1}$}

${ }^{1}$ Toyo Sugar Refining Co., Ltd. Yoto Bldg., 18-20, Nihombashi-Koamicho, Chuo-ku, Tokyo, 103-0016, Japan

*Corresponding author: Yushi Hashizume, Toyo Sugar Refining Co., Ltd. Yoto Bldg., 18-20, Nihombashi-Koamicho, Chuo-ku, Tokyo, 103-0016, Japan

Submission Date: March 17 ${ }^{\text {th }}$, 2021; Acceptance Date: June 9th 2021 ; Publication Date: June 17th, 2021

Please cite this as: Hashizume Y., Tandia M. Suppressive effect of a single dose of monoglucosyl rutin on postprandial blood glucose elevation: A randomized, placebo-controlled, double-blind crossover study. Functional Foods in Health and Disease 2021. 11(6): 270-282. DOI: https://www.doi.org/10.31989/ffhd.v11i6.793

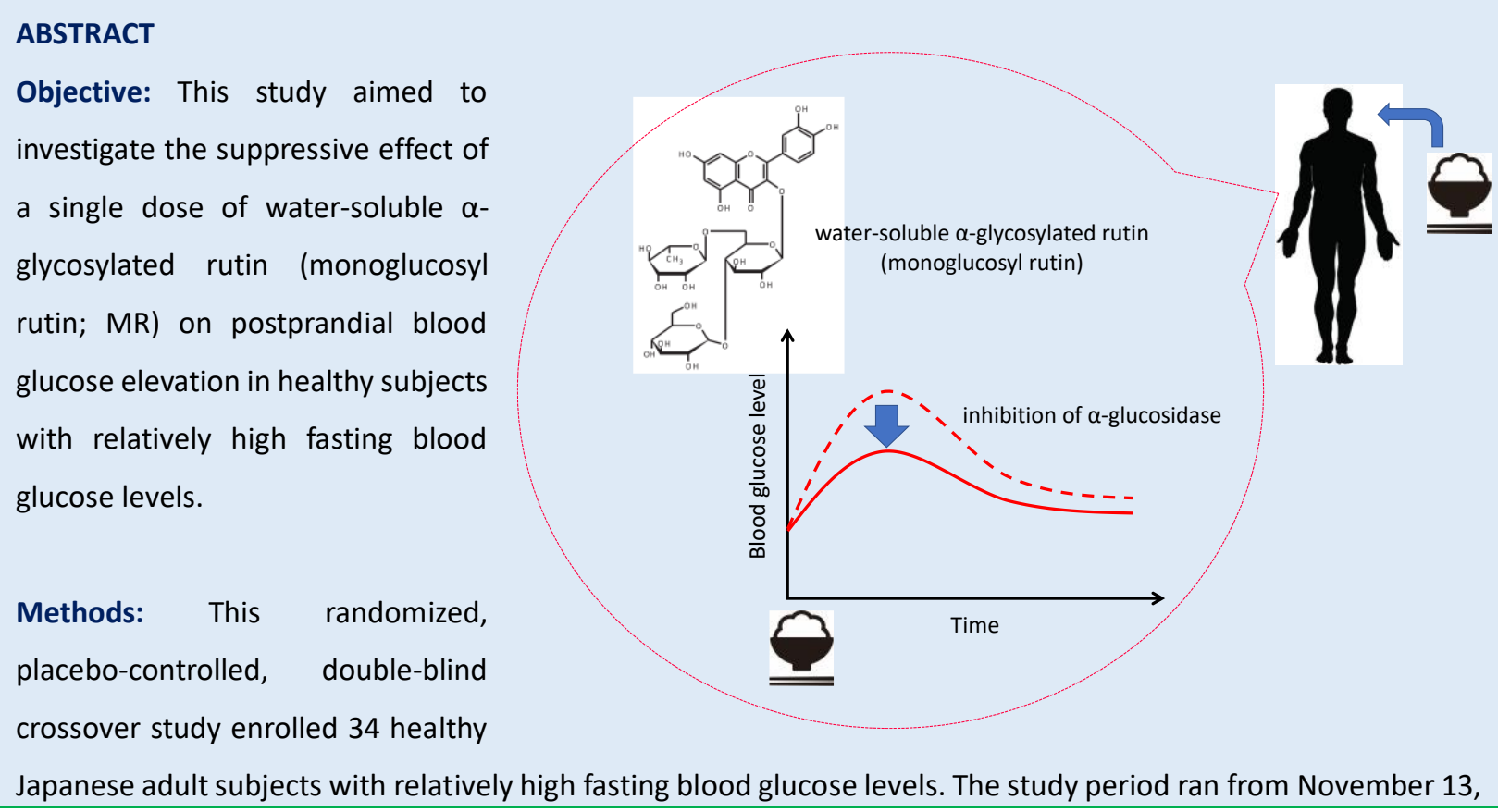


2019, to March 19, 2020. All subjects were randomly allocated to either sequence $A$ or sequence $B$ ( $n=17$ per group) using a computerized random number generator. The washout period was at least one week between periods I and II. In period I, the subjects took either MR or placebo tablets. In period II, subjects took different tablets from the ones they had taken in period $\mathrm{I}$. We evaluated their blood glucose and insulin levels after glucose loading (150 g of cooked rice). The incremental area under the curve (IAUC) of the postprandial blood glucose level was determined as the primary outcome. The blood glucose and insulin levels at maximum (maximum blood concentration; Cmax), each measurement point, and IAUC of the blood insulin level after glucose loading were the secondary outcomes.

Results: Out of 33 subjects, 16 in sequence A (11 men and 5 women, $54.5 \pm 9.8$ years) and 17 in sequence B (9 men and 8 women, $58.8 \pm 9.4$ years) were analyzed as a per-protocol dataset. The glucose IAUC after MR consumption was significantly lower than that of the placebo $(P=0.034)$. Results of the other outcomes were not observed with significant treatment effects. There were no adverse events attributable to the test foods.

Conclusions: We suggest that MR has a suppressive effect on the elevation of postprandial blood glucose in healthy adults with relatively high fasting blood glucose levels.

Trial registration: UMIN-CTR: UMIN000038515.

Foundation: Toyo Sugar Refining Co., Ltd.

Keywords: monoglucosyl rutin, blood glucose level, incremental area under the curve, $\alpha$-amylase, $\alpha$-glucosidase, crossover study

CFFC 2021. This is an Open Access article distributed under the terms of the Creative Commons Attribution 4.0 License (http://creativecommons.org/licenses/by/4.0)

\section{INTRODUCTION}

The prevalence of type II diabetes increases with an unhealthy diet and decreased physical activity. According to a 2019 report from the International Diabetes Federation, one in 11 adults had diabetes[1]. Type II diabetes is a metabolic disorder characterized by hyperglycemia arising from defective insulin signaling. Postprandial hyperglycemia is a major risk factor for type II diabetes; thus, it is important to suppress postprandial blood glucose elevation. Though certain prescriptions such as acarbose are widely used to delay the digestion of ingested carbohydrates in type II diabetes [2-4] by inhibiting $\alpha$-glucosidases, they also cause adverse effects, usually gastrointestinal symptoms such as flatulence, diarrhea, and abdominal pain[2]. It is very important to maintain a healthy diet to control postprandial blood glucose levels before developing diabetes to avoid medication's adverse effects.

Rutin, a flavonoid with significant antioxidant properties, is found in buckwheat, fruits (apricots, berries, cherries, and citrus fruits), vegetables, red 
beans, tea and flowers of certain plants such as the Japanese pagoda tree. Previous studies have reported that rutin shows significant inhibition of $\alpha$-glucosidase in vitro[5], and should suppress the elevation of postprandial blood glucose levels. Single oral administration of rutin reportedly decreased blood glucose levels after glucose loading in rats[6]. Moreover, rutin oral administration for three weeks significantly decreased fasting blood glucose and glycosylated hemoglobin in diabetic rats[7]. Furthermore, supplementing rutin tablets (500 $\mathrm{mg} /$ day) for 60 days has reported significantly decreased fasting blood glucose levels than before consumption in diabetic patients[8].

While these findings suggest rutin's anti-diabetic effects, its application has been limited due to its low water solubility. Toyo Sugar Refining Co., Ltd. has developed the water-soluble $\alpha$-glycosylated rutin (4G$\alpha$-D-glucopyranosyl rutin, monoglucosyl rutin; $M R$ ), which is manufactured from rutin by enzymatic glycosylation. MR is 30,000 times more soluble than common rutin extracts[9]. MR has been used in various products such as beverages, food and functional foods to prevent discoloration, oxidation and lower abdominal visceral. It is also widely used in cosmetics and personal care products to fight reactive free radicals and reactive oxygen species induced by ultraviolet irradiation. MR is metabolized by digestive enzymes in the same way as rutin. However, animal studies have shown that its bioavailability is higher than that of common rutin[10] due to its solubility. Therefore, it is expected that the inhibitory effect of MR on blood glucose elevation can be observed not only in continuous intake as previously reported but also in single-dose intake.

Although the abovementioned studies in diabetic patients and animals indicate that rutin suppresses the elevated fasting or postprandial blood glucose levels, few studies have been examined in healthy subjects. Therefore, in this study, we investigated whether the consumption of MR suppresses the postprandial blood glucose levels in healthy subjects.

\section{METHOD}

Study design: This was a randomized, double-blind, placebo-controlled crossover study. The subjects were allocated to sequences $A$ and $B$ in a 1:1 ratio. The Takara Clinic's ethics committee, Medical Corporation Seishinkai, approved the study protocols on October 25, 2019 (approval ID: 1910-1910-TS01-03-TC). The protocol was registered at the University Hospital Medical Information Network Clinical Trials Registry (UMIN000038515). This study was performed following the Declaration of Helsinki (2013) principles, the ethical guidelines for medical and health research involving human subjects in Japan, and broader medical ethics.

Subjects: The target subjects were healthy Japanese people aged $\geq 20$ years with relatively high fasting blood glucose levels. We excluded the following subjects: (a) subjects who were undergoing medical treatment or had a medical history of malignant tumor, heart failure, or myocardial infarction; (b) subjects who had a pacemaker or an implantable cardioverter defibrillator; (c) subjects who were undergoing treatment for any of the following chronic diseases: arrhythmia, liver disease, kidney disease, cerebrovascular disease, rheumatism, diabetes mellitus, hyperlipidemia, hypertension, or any other chronic diseases; (d) subjects who take "Foods for Specified Health Uses," "Foods with Functional Claims," or other functional food/beverage daily; (e) subjects who were currently taking medicines (including herbal medicines) and supplements; (f) subjects who were allergic to medicines and/or the test-food-related products; (g) subjects who were pregnant, breastfeeding, and planning to become pregnant; (h) 
subjects who had been enrolled in other clinical studies within the last three months before the agreement to participate in this study or plan to participate another study during this study; and (i) subjects who were judged as ineligible to participate in the study by the physician. All subjects enrolled through the website (https://www.go106.jp/) operated by ORTHOMEDICO Inc. (Tokyo, Japan). The study protocols were comprehensively explained to all subjects at the office of ORTHOMEDICO Inc. All subjects gave their informed consent before they participated in the study. No sponsors or members of the funding companies participated in the study. The assessment was conducted at Takara Clinic (Medical Corporation Seishinkai, Tokyo, Japan).

Intervention: The active tablet contained MR ( $\alpha$ G Rutin $\mathrm{PS}^{\mathrm{TM}}$ ), and the placebo was made of dextrin. Test foods were provided by Toyo Sugar Refining Co., Ltd. (Tokyo, Japan). The ethics committee declared that both tablets were identical in color, odor, and flavor. As for the glucose loading diet, subjects took Sato No Gohan (150 g; retort cooked rice) (Sato Foods Industries Co., Ltd., Niigata, Japan) during periods I and II. During period I, we asked all subjects to take tablet of either MR (200mg as MR) or placebo $30 \mathrm{~min}$ before consuming the loading diet. During period II, subjects took either MR or placebo, which they had not taken in period I. There was a washout period of at least one week between the two periods. The subjects assigned to Sequence A took the MR and the placebo in periods I and II, respectively. Meanwhile, those assigned to Sequence $B$ took the placebo and MR in periods I and II, respectively.

Outcomes: The study schedule is shown in Table 1. Subjects visited the clinic and underwent examinations before consuming test food $(\mathrm{Scr})$ and during periods I and II. All physical examinations were conducted at the
Takara clinic. Urinalysis and hematological analysis were conducted at LSI Medience Corporation (Tokyo, Japan).

(1) Primary outcome: Incremental area under the curve (IAUC) of postprandial blood glucose level Approximately $2 \mathrm{~mL}$ of venous blood was collected from subjects $30,60,90$, and 120 min after consuming the loading diet.

(2) Secondary outcomes: Maximum blood concentration $\left(\mathrm{C}_{\max }\right)$ of the blood glucose and blood glucose levels at each measurement point after glucose loading

Measurements were conducted in the same way as the primary outcome.

(3) Secondary outcomes: IAUC of blood insulin level, $\mathrm{C}_{\max }$ of blood insulin, and blood insulin levels at each measurement point after glucose loading Approximately $6 \mathrm{~mL}$ of venous blood was collected from subjects $30,60,90$, and 120 min after consuming the loading diet.

\section{(4) Safety evaluation}

We conducted a physical examination, urinalysis, and hematological analysis at Scr. Systolic and diastolic blood pressures and pulse rate were also measured during periods I and II.

The subjects' height, weight, BMI, body fat percentage, temperature, systolic and diastolic blood pressures, and pulse rate were measured as the physical examination parameters. The height was measured at the briefing to calculate their BMI.

Hematological tests and urinalysis were conducted as well.

Furthermore, we asked the subjects to complete a medical questionnaire to determine their health status at each assessment point. 
Table 1. Schedule of enrollment, intervention, and assessments.

\begin{tabular}{|c|c|c|c|c|c|c|c|}
\hline & \multicolumn{4}{|c|}{ Screening } & \multicolumn{3}{|c|}{ Period } \\
\hline & Briefing & Scr & Enrollment & Allocation & I & Washout & II \\
\hline \multicolumn{8}{|l|}{ ENROLLMENT: } \\
\hline \multicolumn{8}{|l|}{ Eligibility screen } \\
\hline \multicolumn{8}{|l|}{ Informed consent } \\
\hline \multicolumn{8}{|l|}{ Allocation } \\
\hline \multicolumn{8}{|l|}{ INTERVENTIONS: } \\
\hline \multicolumn{8}{|l|}{ MR or Placebo } \\
\hline \multicolumn{8}{|l|}{ Loading food } \\
\hline \multicolumn{8}{|l|}{ ASSESSMENTS: } \\
\hline \multicolumn{8}{|l|}{ Outcome } \\
\hline \multicolumn{8}{|l|}{ Physical examination I } \\
\hline \multicolumn{8}{|l|}{ Physical examination II } \\
\hline \multicolumn{8}{|l|}{ Urinalysis } \\
\hline \multicolumn{8}{|l|}{ Hematological analysis } \\
\hline Medical questionnaire & & 0 & & & D & & 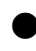 \\
\hline
\end{tabular}

Closed circles ( ) display the execution timing of each items.

Scr, examination before consumption of test foods.

Physical examination I, measurement of height, weight, BMI, body fat percentage, and temperature.

Physical examination II, measurement of systolic and diastolic blood pressures and pulse rate. 
Sample size: To our knowledge, no clinical studies have measured the IAUC of blood glucose in healthy adult subjects taking MR-containing tablets. Therefore, the difference between the consumption of MR and the consumption of placebo was hypothetically moderate in this study. According to Cohen (1992)[11], the effect size (d) was determined to be 0.50 . The number of cases was calculated using a statistical significance level $(\alpha)$ of 0.05 , statistical power $(1-\beta)$ of 0.80 , and an individual difference index of 1.0. The required number of cases was 34 (17 in each sequence).

Selection, randomization, and blinding: Among the 241 subjects who provided informed consent, 34 were considered eligible by the physician. The selection criterion was to have a fasting blood glucose level between $\geq 110 \mathrm{mg} / \mathrm{dL}$ and $<126 \mathrm{mg} / \mathrm{dL}$ or glucose concentration two hours after $75-\mathrm{g}$ oral glucose tolerance [Trelan-G ${ }^{\circledR}$ solution $(75$ g) (EY Pharmaceuticals Co., Ltd., Tokyo, Japan)] test (OGTT) value between $\geq 140 \mathrm{mg} / \mathrm{dL}$ and $<200 \mathrm{mg} / \mathrm{dL}$ at Scr. The oral glucose tolerance loading was conducted at Scr only. The test foods were provided by the sponsor of the contract research organization. After the test foods were confirmed to be indistinguishable and data at Scr were inputted and confirmed, the test foods administrator, who is a member of the contract research organization, gave the code of the test foods to an allocation controller who was not directly involved in this study. This study's allocation was a stratified random assignment using age, gender, and a fasting blood glucose level at Scr as allocation adjustment factors. The subjects were randomly allocated to either sequence $A$ or sequence $B(n=17$ each) by the allocation controller according to a computergenerated randomization list. The allocation table with the coded sequences was provided only to the test foods administrator, who provided the test foods to each subject according to the table. The allocation controller locked the allocation table until the key opening day. The sponsors, principal investigator, sub- investigators, entire contract research organization staff (i.e., the director of the study, the director of study conduction, the person in charge of monitoring, the director and staff of statistical analysis, and the administrator of the test foods), medical institution staff, institutional review board members, contract laboratory, and others who were associated with this study were not aware of the group assignments or involved in allocation.

Statistical analysis: All statistical analyses were performed with two-sided tests, and the significance level was set at 5\%. SPSS ver. 23.0 (IBM Japan, Ltd., Tokyo, Japan) for Windows was used for analysis. The measured values in physical examination, urinalysis, and hematological analysis at Scr were presented using the mean and standard deviation (SD). These data were analyzed using Student's $t$-test. Meanwhile, gender and the results of hematological analysis and urinalysis were set to a code, i.e., 1 was defined as falling within the normal range (for man), whereas 0 was defined as outside the normal range (or woman). These data were expressed as the number of subjects ( $n$ ) and were analyzed between groups using the chi-square test at each sequence. Measured values of blood glucose, insulin levels, systolic and diastolic blood pressures, and pulse rate were presented using the mean, SD, estimated marginal means (EMM), standard error (SE) based on EMM, and the $95 \%$ confidence intervals $(\mathrm{Cl})$. These data were analyzed using a general linear mixed model analysis of variance (ANOVA) with group factors (sequence $A$ or sequence $B$ ), subjects nested in the group, study period (the periods I and II), and test food (MR tablets and placebo) to calculate the period effect, carryover effect, and treatment effect. The principal investigator also assessed the safety endpoints, ensuring that no medically problematic changes associated with the consumption of the test foods occurred. All combinations were compared, and data were not considered in the multiplicity. 


\section{RESULTS}

Analysis set: Figure 1 shows the flowchart of this study. The target subjects were healthy Japanese aged $\geq 20$ years with moderate hyperglycemia. We recruited them from November 8, 2019, to February 26, 2020. This study was conducted from November 13, 2019, to March 19, 2020.
Out of 241 subjects, 34 were eligible and were randomly divided into either sequence $A$ or sequence $B$ ( $n=17$ each). During the study period, subjects who had eaten within six hours before blood collection were excluded from the statistical analysis. Therefore, 33 subjects (20 men and 13 women) were included in the statistical analysis on the per-protocol set. Table 2 shows the subjects' background information.

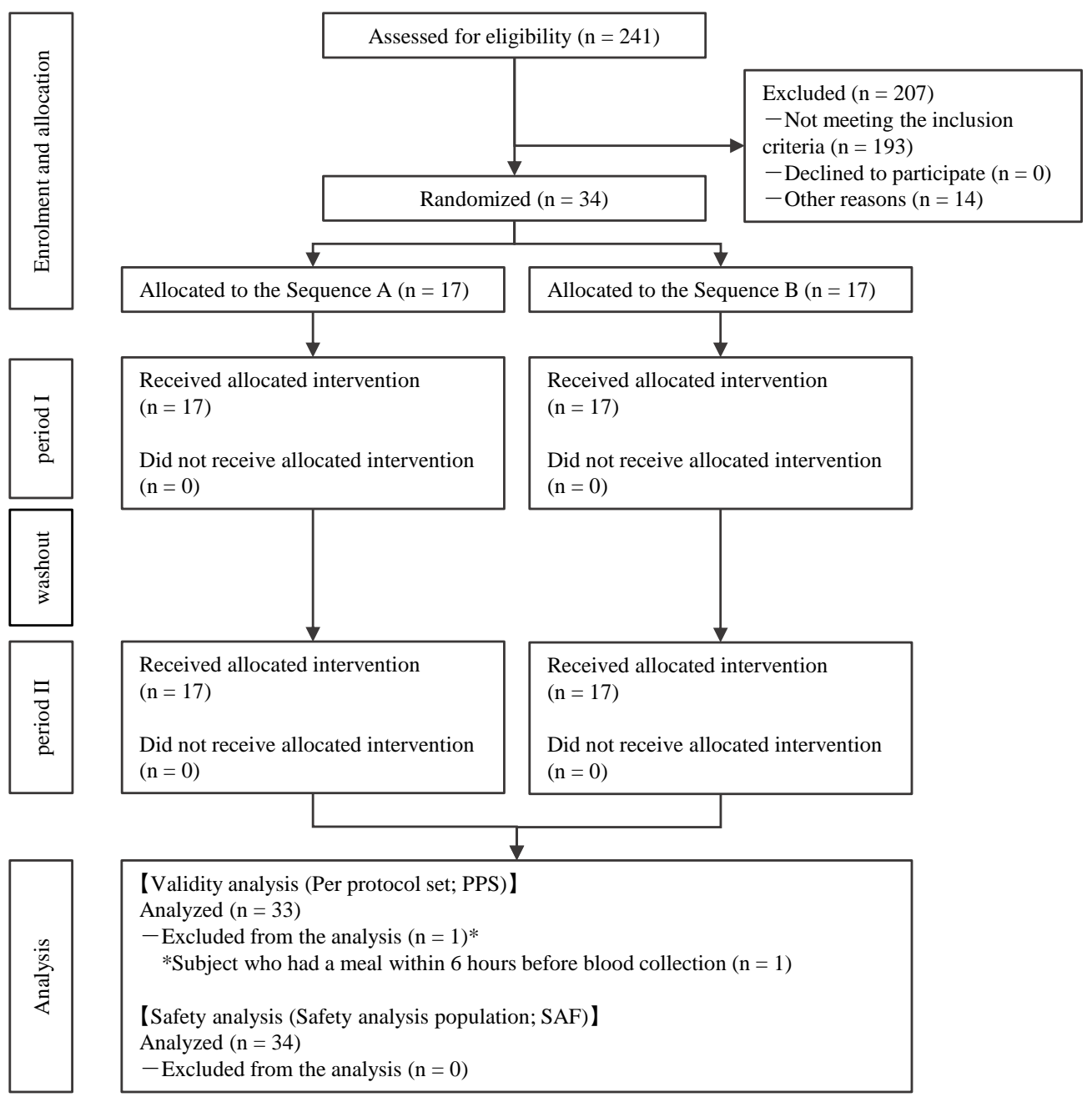

Figure 1. Flowchart of the study procedure

Blood glucose levels after glucose loading: Figure 2 shows the results of IAUC. Table 3 shows the results of $C_{\max }$ and blood glucose levels at each measurement after glucose loading. IAUCs upon consumption of MR and placebo were $94.5 \pm 38.0 \mathrm{U} / \mathrm{L}$ and $106.3 \pm 33.9 \mathrm{U} / \mathrm{L}$, respectively. The treatment effect on IAUC upon consumption of MR was significant $(P=0.034)$, and the average effect was lower than that in placebo (carryover effect, $P=0.761$, period effect, $P=0.684$ ).

Blood insulin levels after glucose loading: Table 4 shows the results of IAUC, $C_{\max }$, and blood insulin levels 
at each measurement after glucose loading. The carryover effects on blood insulin levels 60 and $90 \mathrm{~min}$ after a glucose load were significant $(P=0.048$, $P=0.039$, respectively).
Physical examination: Table 5 shows the measured values of systolic blood pressure, diastolic blood pressure, and pulse rate. There were no significant differences in the results between the interventions

Table 2. Background information on subjects assigned to this study

\begin{tabular}{|c|c|c|c|c|c|c|c|c|}
\hline & \multirow[t]{2}{*}{ Unit } & \multicolumn{2}{|c|}{$\begin{array}{l}\text { All subjects } \\
\qquad(n=34)\end{array}$} & \multicolumn{2}{|c|}{$\begin{array}{l}\text { Sequence A } \\
\qquad(n=17)\end{array}$} & \multicolumn{2}{|c|}{$\begin{array}{l}\text { Sequence } B \\
\qquad(n=17)\end{array}$} & \multirow[t]{2}{*}{$\begin{array}{c}P \\
\text { value }\end{array}$} \\
\hline & & Mean & SD & Mean & SD & Mean & SD & \\
\hline Age & years & 56.6 & 9.7 & 54.5 & 9.8 & 58.8 & 9.4 & 0.208 \\
\hline \multicolumn{9}{|l|}{ Physical examination } \\
\hline Height & $\mathrm{cm}$ & 163.7 & 7.1 & 165.1 & 6.8 & 162.3 & 7.3 & 0.246 \\
\hline Weight & $\mathrm{kg}$ & 67.3 & 11.6 & 70.4 & 11.6 & 64.1 & 11.1 & 0.114 \\
\hline $\mathrm{BMI}$ & $\mathrm{kg} / \mathrm{m}^{2}$ & 25.0 & 3.6 & 25.7 & 3.5 & 24.3 & 3.6 & 0.231 \\
\hline Body fat percentage & $\%$ & 26.6 & 7.1 & 26.5 & 8.1 & 26.8 & 6.1 & 0.906 \\
\hline Systolic blood pressure & $\mathrm{mmHg}$ & 129.2 & 14.5 & 127.7 & 12.9 & 130.7 & 16.1 & 0.550 \\
\hline Diastolic blood pressure & $\mathrm{mmHg}$ & 83.9 & 11.5 & 81.4 & 11.0 & 86.5 & 11.8 & 0.200 \\
\hline Pulse rate & bpm & 74.1 & 9.8 & 73.9 & 12.3 & 74.4 & 6.9 & 0.891 \\
\hline Body temperature & ${ }^{\circ} \mathrm{C}$ & 36.2 & 0.3 & 36.3 & 0.4 & 36.1 & 0.3 & 0.107 \\
\hline
\end{tabular}

The data are presented as the mean and standard deviation (SD).

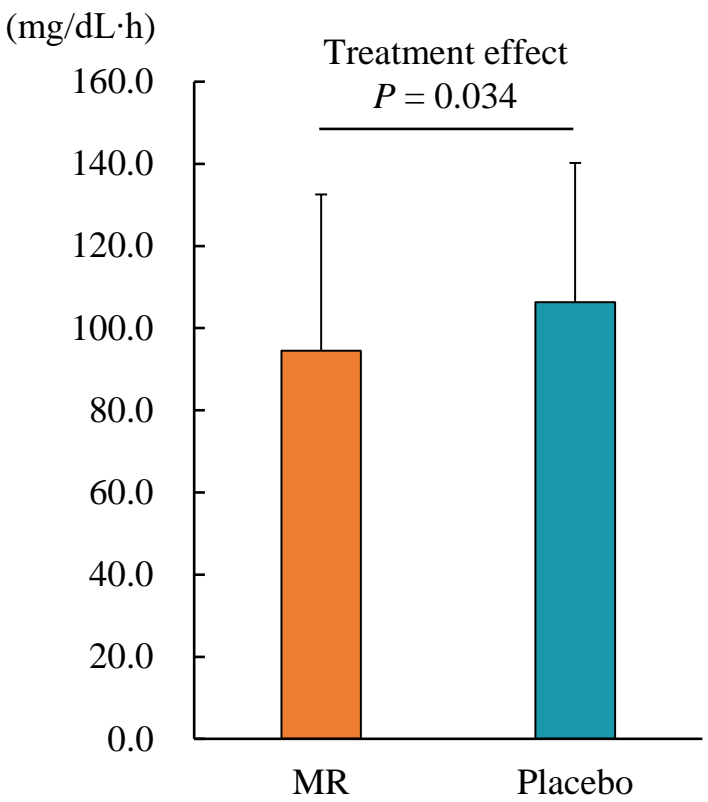

Figure 2. IAUC of the postprandial blood glucose level. The data are presented as the mean and standard deviation (SD). EMM of the difference value obtained by subtracting the placebo from MR was -11.752 (SE 5.286, 95\% CI: -22.533 to -0.972 ). 
Table 3. Blood glucose level after glucose loading.

\begin{tabular}{|c|c|c|c|c|c|c|c|c|c|c|c|c|}
\hline & \multirow[t]{2}{*}{ Unit } & \multicolumn{2}{|c|}{ MR } & \multicolumn{2}{|c|}{ Placebo } & \multicolumn{4}{|c|}{$\Delta$ MR-Placebo } & \multicolumn{3}{|c|}{$P$ value } \\
\hline & & Mean & SD & Mean & SD & EMM & SE & $95 \% \mathrm{CI}-$ & $95 \% \mathrm{CI}+$ & $\begin{array}{c}\text { Treatment } \\
\text { effect }\end{array}$ & $\begin{array}{c}\text { Carryover } \\
\text { effect }\end{array}$ & $\begin{array}{l}\text { Period } \\
\text { effect }\end{array}$ \\
\hline $\mathbf{C}_{\max }$ & $\mathrm{mg} / \mathrm{dL}$ & 166.2 & 35.9 & 173.5 & 29.2 & -7.265 & 3.775 & -14.965 & 0.435 & 0.064 & 0.760 & 0.394 \\
\hline \multicolumn{13}{|l|}{ Glucose level } \\
\hline $\begin{array}{l}\text { before taking } \\
\text { the test food }\end{array}$ & $\mathrm{mg} / \mathrm{dL}$ & 94.8 & 15.2 & 94.5 & 15.4 & 0.254 & 0.915 & -1.612 & 2.120 & 0.783 & 0.972 & 0.144 \\
\hline $\begin{array}{l}\mathbf{3 0} \text { minutes } \\
\text { after a glucose } \\
\text { load }\end{array}$ & $\mathrm{mg} / \mathrm{dL}$ & 142.8 & 22.1 & 146.3 & 21.5 & -3.469 & 2.227 & -8.010 & 1.072 & 0.129 & 0.195 & 0.514 \\
\hline $\begin{array}{l}60 \text { minutes } \\
\text { after a glucose } \\
\text { load }\end{array}$ & $\mathrm{mg} / \mathrm{dL}$ & 159.5 & 39.4 & 168.9 & 32.0 & -9.346 & 4.733 & -18.998 & 0.307 & 0.057 & 0.683 & 0.339 \\
\hline $\begin{array}{l}90 \text { minutes } \\
\text { after a glucose } \\
\text { load }\end{array}$ & $\mathrm{mg} / \mathrm{dL}$ & 150.9 & 40.1 & 158.6 & 34.6 & -7.585 & 4.308 & -16.371 & 1.201 & 0.088 & 0.938 & 0.396 \\
\hline $\begin{array}{l}120 \text { minutes } \\
\text { after a glucose } \\
\text { load }\end{array}$ & $\mathrm{mg} / \mathrm{dL}$ & 133.7 & 33.3 & 139.4 & 33.6 & -5.623 & 3.771 & -13.314 & 2.068 & 0.146 & 0.268 & 0.369 \\
\hline
\end{tabular}

The data are presented as the mean standard deviation (SD), estimated marginal means (EMM), standard error (SE) based on EMM, and the $95 \%$ confidence intervals $(95 \% \mathrm{CI}-$, $95 \% \mathrm{CI}+)$.

$\Delta \mathrm{MR}$ - Placebo, the difference value obtained by subtracting the placebo from MR. 
Table 4. Blood insulin level after glucose loading.

\begin{tabular}{|c|c|c|c|c|c|c|c|c|c|c|c|c|}
\hline & \multirow[t]{2}{*}{ Unit } & \multicolumn{2}{|c|}{ MR } & \multicolumn{2}{|c|}{ Placebo } & \multicolumn{4}{|c|}{$\Delta$ MR-Placebo } & \multicolumn{3}{|c|}{$P$ value } \\
\hline & & Mean & SD & Mean & SD & EMM & SE & $95 \% \mathrm{CI}-$ & $95 \% \mathrm{CI}+$ & $\begin{array}{c}\text { Treatment } \\
\text { effect }\end{array}$ & $\begin{array}{c}\text { Carryover } \\
\text { effect }\end{array}$ & $\begin{array}{l}\text { Period } \\
\text { effect }\end{array}$ \\
\hline IAUC & $\mu \mathrm{U} / \mathrm{mL} \cdot \mathrm{h}$ & 33.5 & 19.3 & 35.4 & 20.0 & -1.940 & 1.477 & -4.953 & 1.072 & 0.199 & 0.066 & 0.971 \\
\hline $\mathbf{C}_{\max }$ & $\mu \mathrm{U} / \mathrm{mL}$ & 33.0 & 16.8 & 35.3 & 17.4 & -2.213 & 1.271 & -4.804 & 0.379 & 0.092 & 0.087 & 0.321 \\
\hline \multicolumn{13}{|l|}{ Insulin level } \\
\hline $\begin{array}{l}\text { before taking } \\
\text { the test food }\end{array}$ & $\mu \mathrm{U} / \mathrm{mL}$ & 6.4 & 3.2 & 6.2 & 3.4 & 0.240 & 0.297 & -0.365 & 0.845 & 0.425 & 0.129 & 0.459 \\
\hline $\begin{array}{l}\mathbf{3 0} \text { minutes } \\
\text { after a glucose } \\
\text { load }\end{array}$ & $\mu \mathrm{U} / \mathrm{mL}$ & 21.6 & 11.4 & 21.2 & 11.8 & 0.366 & 0.909 & -1.488 & 2.221 & 0.690 & 0.128 & 0.325 \\
\hline $\begin{array}{l}60 \text { minutes } \\
\text { after a glucose } \\
\text { load }\end{array}$ & $\mu \mathrm{U} / \mathrm{mL}$ & 25.5 & 13.9 & 27.5 & 13.7 & -1.972 & 1.630 & -5.296 & 1.352 & 0.235 & $0.048 *$ & 0.620 \\
\hline $\begin{array}{l}90 \text { minutes } \\
\text { after a glucose } \\
\text { load }\end{array}$ & $\mu \mathrm{U} / \mathrm{mL}$ & 29.1 & 15.4 & 29.8 & 18.2 & -0.643 & 1.745 & -4.202 & 2.916 & 0.715 & $0.039 *$ & 0.694 \\
\hline $\begin{array}{l}120 \text { minutes } \\
\text { after a glucose } \\
\text { load }\end{array}$ & $\mu \mathrm{U} / \mathrm{mL}$ & 26.3 & 16.6 & 27.9 & 17.7 & -1.583 & 1.543 & -4.730 & 1.563 & 0.313 & 0.170 & 0.925 \\
\hline
\end{tabular}

The data are presented as the mean standard deviation (SD), estimated marginal means (EMM), standard error (SE) based on EMM, and the 95\% confidence intervals (95\%CI-, $95 \% \mathrm{CI}+)$.

$\Delta \mathrm{MR}$ - Placebo, the difference value obtained by subtracting the placebo from MR.

$* P<0.05$ vs. Placebo 
Table 5. Physical examination.

\begin{tabular}{|c|c|c|c|c|c|c|c|c|c|c|c|c|}
\hline & Unit & M & & Plac & bo & & $\Delta \mathrm{MR}$ & - Placebo & & & $P$ value & \\
\hline & & Mean & SD & Mean & SD & EMM & SE & $95 \% \mathrm{CI}-$ & $95 \% \mathrm{CI}+$ & $\begin{array}{c}\text { Treatment } \\
\text { effect }\end{array}$ & $\begin{array}{c}\text { Carryover } \\
\text { effect }\end{array}$ & $\begin{array}{r}\text { Period } \\
\text { effect }\end{array}$ \\
\hline Systolic blood pressure & $\mathrm{mmHg}$ & 125.4 & 13.6 & 123.6 & 14.6 & 1.824 & 1.820 & -1.883 & 5.530 & 0.324 & 0.103 & 0.260 \\
\hline Diastolic blood pressure & $\mathrm{mmHg}$ & 81.0 & 10.6 & 80.9 & 11.2 & 0.147 & 1.143 & -2.181 & 2.475 & 0.898 & 0.263 & 0.139 \\
\hline Pulse rate & bpm & 74.4 & 10.5 & 74.6 & 11.3 & -0.176 & 1.175 & -2.570 & 2.217 & 0.882 & 0.656 & 0.638 \\
\hline
\end{tabular}

The data are presented as the mean standard deviation (SD), estimated marginal means (EMM), standard error (SE) based on EMM, and the $95 \%$ confidence intervals ( $95 \%$ CI-, $95 \% \mathrm{CI}+$ ).

$\Delta \mathrm{MR}-$ Placebo, the difference value obtained by subtracting the placebo from MR. 


\section{DISCUSSION}

We investigated MR's suppressive effect on the elevation of postprandial blood glucose in healthy subjects with relatively high fasting blood glucose levels. The primary outcome, IAUC of postprandial blood glucose level, was observed of significant treatment effect $(P=0.034)$, and the value upon consumption of MR was lower than that of placebo. Secondary outcomes, including $\mathrm{C}_{\max }$ of glucose and blood glucose levels at $60 \mathrm{~min}$ and $90 \mathrm{~min}$ after a glucose load, tended to be different in treatment effect $(P=0.064, P=0.057$, and $P=0.088$, respectively), and each value upon consumption of MR was lower than that upon consumption of placebo. While treatment effects were observed in the above results, carryover effects were observed in the blood insulin levels at $60 \mathrm{~min}$ and $90 \mathrm{~min}$ after the loading diet. A carryover effect means that the firstperiod treatment affects the response to the secondperiod treatment so that our results could not provide evidence of an effect of MR on the blood insulin levels.

In this study, subjects were asked to take milled rice as glucose loading diet. It contains more than $75 \%$ of carbohydrates[12], mostly starch, such as a simple polysaccharide composed of amylose and amylopectin[13]. They are hydrolyzed to glucose monosaccharides, maltose disaccharides, and oligosaccharides by $\alpha$-amylase. Disaccharides are finally hydrolyzed by $\alpha$-glucosidase to glucose. Monosaccharides such as glucose are absorbed into the bloodstream from the intestinal tract[13]. According to the finding that rutin inhibits the activity of $\alpha$-amylase and $\alpha$-glucosidase[5], our results suggest consuming the test food containing MR suppresses postprandial blood glucose elevation inhibiting the activity of a glucose-hydrolytic enzyme and absorption of glucose.

A possible reason for the insignificant change in the blood insulin levels in this study is an insulinindependent mechanism to suppress the elevated postprandial blood glucose levels. Glucose transporter 2 (GLUT2), the major glucose transporter mainly expressed in hepatocytes, acts independently of insulin[14][15]. GLUT2 is thought to respond to blood glucose concentration changes and alter the rate of glucose uptake by hepatocytes[14]. One study suggested that rutin increases GLUT2 protein expression and promotes increasing glucose uptake by hepatocytes, resulting in the alleviation of insulin resistance induced by high blood glucose levels[14].

While we conducted a single dose administration of MR, other studies have demonstrated that a continuous administration of rutin decreases the fasting blood glucose levels[7,8]; thus, we should also investigate the anti-diabetic effects of the continuous administration of $M R$ in further studies. The continuous administration of rutin might play a positive role in carbohydrate metabolism by activating gluconeogenesis enzymes and has a protective effect on the pancreas in diabetic rats[16]. In future studies, measuring the markers for pancreatic function and glucose metabolism might lead to some interesting discoveries for elucidating the mechanism of the hypoglycemic effect of MR. Additionally, clinical studies on the efficacy of MR to inhibit or lower the formation of dietary advance glycation end-products are needed.

\section{CONCLUSION}

This study investigated the suppressive effect of MR on the elevation of postprandial blood glucose in healthy subjects with relatively high fasting blood glucose levels. We found that MR significantly reduces postprandial blood glucose levels.

Abbreviations: IAUC: incremental area under the curve, $C_{\text {max }}$ : maximum blood concentration, $\mathrm{BMI}$ : 
body mass index, OGTT: oral glucose tolerance test, GLUT2: glucose transporter 2, SD: standard deviation, SE: standard error, EMM: estimated marginal mean, ANOVA: analysis of variance

Competing Interests: The sponsor of the present study, Toyo Sugar Refining Co., Ltd., assigned ORTHOMEDICO Inc. to conduct the study. Yushi Hashizume and Mahamadou Tandia were affiliated with Toyo Sugar Refining Co., Ltd.

Authors' Contributions: Yushi Hashizume drafted the manuscript. Yushi Hashizume and Mahamadou Tandia designed the study, and interpreted the results.

Acknowledgments and Funding: The authors would like to thank all subjects and staff who participated in this study. We also thank Tsuyoshi Takara (MD) and ORTHOMEDICO Inc. for technical assistance regarding the study. Finally, special appreciation is extended to Kenichi Kakino of the Evaluation Center of Health and Nutrition Inc. for his contributions.

Toyo Sugar Refining Co., Ltd funded the study implementation and manuscript writing.

\section{REFERENCES}

1. International Diabetes Federation: IDF Diabetes Atlas 9th edition 2019. 2019,

2. Mclver LA, Tripp J, Brooklyn T: Acarbose. StatPearls 2020,

3. Yee $\mathrm{H}$, Fong $\mathrm{N}$ : A review of the safety and efficacy of acarbose in diabetes mellitus. Pharmacotherapy 1996, 16 (5): 792-805.

4. Holman RR, Cull CA, Turner RC: A randomized doubleblind trial of acarbose in type 2 diabetes shows improved glycemic control over 3 years (U.K. Prospective Diabetes Study 44). Diabetes Care 1999, 22 (6): 960-964.

5. Dubey S, Ganeshpurkar A, Ganeshpurkar A, Bansal D, Dubey N: Glycolytic enzyme inhibitory and antiglycation potential of rutin. Futur J Pharm Sci 2017, 3 (2): 158162.

6. Pereira D, Cazarolli L, Lavado C, Mengatto V, Figueiredo
M, Guedes A, Pizzolatti M, Silva F: Effects of flavonoids on $\alpha$-glucosidase activity: Potential targets for glucose homeostasis. Nutrition 2011, 27 (11-12): 1161-1167.

7. Niture $\mathrm{N}$, Ansari A, Naik S: Anti-hyperglycemic activity of Rutin in streptozotocin-induced diabetic rats: An effect mediated through cytokines, antioxidants and lipid biomarkers. Indian J Exp Biol 2014, 52 (7): 720-727.

8. Sattanathan K, Dhanapal CK, Umarani R, Manavalan R: Beneficial health effects of rutin supplementation in patients with diabetes mellitus. J Appl Pharm Sci 2011, 1 (8): 227-231.

9. Suzuki Y, Suzuki K: Enzymatic formation of 4G-.ALPHA.D-glucopyranosyl-rutin. Agric Biol Chem 1991, 55 (1): 181-187.

10. Shimoi K, Yoshizumi K, Kido T, Usui $\mathrm{Y}$, Yumoto $\mathrm{T}$ : Absorption and Urinary Excretion of Quercetin, Rutin, and $\alpha$ G-Rutin, a Water Soluble Flavonoid, in Rats. J Agric Food Chem 2003, 51 (9): 2785-2789.

11. Cohen J: A power primer. Psychol Bull 1992, 112 (1): 155-9.

12. Ministry of education, culture, sports science and technology-J: STANDARD TABLES OF FOOD COMPOSITION IN JAPAN -2015- (Seventh Revised Edition) -Available Carbohydrates, Polyols and Organic Acids -. 2015.

13. Shiozaki Y, Miyamoto K: 1-1. Carbohydrate (In Japanese). in Guideline for Clinical Nutrition, 2014: 2-6.

14. Lee CC, Hsu WH, Shen SR, Cheng YH, Wu SC: Fagopyrum tataricum (Buckwheat) improved high-glucose-induced insulin resistance in mouse hepatocytes and diabetes in fructose-rich diet-induced mice. Exp Diabetes Res 2012.

15. Hsu C-Y, Shih H-Y, Chia Y-C, Lee $\mathrm{C}-\mathrm{H}$, Ashida H, Lai $\mathrm{Y}-\mathrm{K}$, Weng C-F: Rutin potentiates insulin receptor kinase to enhance insulin-dependent glucose transporter 4 translocation. Mol Nutr Food Res 2014, 58 (6): 11681176.

16. Stanley Mainzen Prince $P$, Kamalakkannan N: Rutin improves glucose homeostasis in streptozotocin diabetic tissues by altering glycolytic and gluconeogenic enzymes. J Biochem Mol Toxicol 2006, 20 (2): 96-102. 